Proceedings of the 26th Annual International Conference of the IEEE EMBS

San Francisco, CA, USA • September 1-5, 2004

\title{
Adaptive Assistance for Guided Force Training in Chronic Stroke
}

\author{
L. E. Kahn ${ }^{1,2}$, W. Z. Rymer ${ }^{1,2,3}$, D. J. Reinkensmeyer ${ }^{2,4}$ \\ ${ }^{1}$ Department of Biomedical Engineering, Northwestern University, IL, USA \\ ${ }^{2}$ Sensory Motor Performance Program, Rehabilitation Institute of Chicago, IL, USA \\ ${ }^{3}$ Department of Phys. Med. and Rehabilitation, Northwestern University Feinberg School of Medicine, Chicago, IL, USA \\ ${ }^{4}$ Department of Mechanical and Aerospace Engineering, University of California - Irvine, CA, USA
}

Abstract - This paper describes a novel form of robotic therapy for the upper extremity in chronic stroke. Based on previous results, we hypothesized that a training task that encourages subjects to consciously guide endpoint forces generated by the hemiparetic arm will result in significant gains in functional ability of the arm, superior to more conventional methods of therapy. In addition, since stroke survivors present with varying degrees of arm movement ability, we developed an adaptive algorithm that tailors the amount of assistance provided in completing the guided force training task. The algorithm adapts a coefficient for velocitydependent assistance based on measured movement speed, on a trial-to-trial basis. The training algorithm has been implemented with a simple linear robotic device called the ARM Guide. One participant completed a two month training program with the adaptive algorithm, resulting in significant improvements in the performance of functional tasks. control

Keywords - Robotics, Stroke, Rehabilitation, Adaptive

\section{INTRODUCTION}

Robotic devices have been investigated as tools in upper extremity rehabilitation for chronic stroke survivors [1-4]. The first device used as a therapeutic tool, the MITMANUS, demonstrated that arm function in stroke survivors can benefit from interacting with an actuated planar device in the subacute stages of recovery [5]. Subsequently, two devices, MIME [4] and ARM Guide [3], expanded the investigations of therapeutic applications of robots into the chronic stroke population. These two studies verified that repetitive interaction with a mechanical device can result in improved performance of functional tasks. However, the outcomes of these two studies differed in that the reach extents of participants after eight weeks of training was different between the two devices. Participants in the MIME study were able to reach farther towards a target during unsupported and unguided movement, while users of the ARM Guide showed no change. After examining the differences between the methods in the two studies, we hypothesized that the fact that subjects consciously guided endpoint forces towards the reaching target with MIME may have been most responsible for the difference [6]. We decided, therefore, to test this "guided force" method with the ARM Guide

One difficulty that arises in designing such a training paradigm in a stroke population is that participants exhibit a wide range of arm impairment levels. Because of this, some subjects are able to move through a large range of motion at a high velocity while others have severe range and velocity limitations. Adapting the level of assistance on a patientspecific basis is desirable because it would allow each patient to achieve as normal-as-possible movement, but with the least amount of assistance possible. This paper reports the development of a robotic movement training algorithm that couples guided force training and adaptive assistance.

\section{Methodology}

The new training method was implemented with the ARM Guide (Fig. 1). The device consists of a servo motor (M) controlling the position of the subject's arm (A), which is coupled to the device through a handpiece $(\mathrm{H})$, along a linear track in the reaching direction. Two other degrees of freedom about the yaw and elevation axes (arrows) allow subjects to practice reaching movements to different areas of the workspace. A single trial, however, is performed with these two axes locked and movement only in the reaching direction. A six-axis load cell $(\mathrm{F})$ reports the forces and torques at the interface between the subject and the device. The device is statically counterbalanced (C) so that it does not gravitationally load the arm. For a more detailed description, see [3].

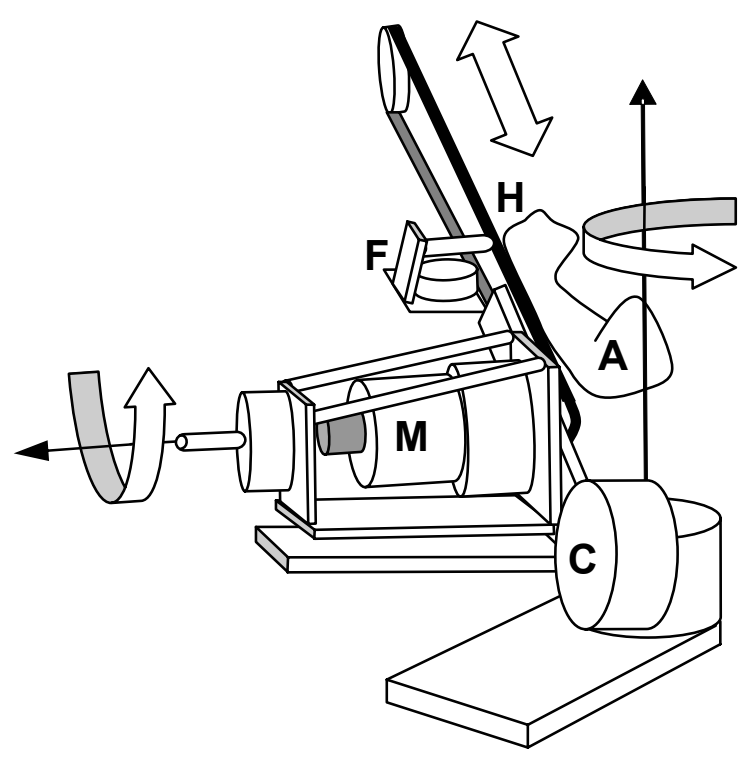

Fig. 1. Diagram of the ARM Guide 
The objective of guided force training is to promote conscious shaping of endpoint forces in the hemiparetic arm. Thus, a method was needed to make the subject aware of the forces they were generating. While movement was only allowed along the linear track of the ARM Guide, the load cell was used to record misdirected or "off-axis" forces generated by the user. During a single trial the user attempts to reach forward at a comfortable speed to the end of his or her range of motion. Throughout the movement, the load cell is monitored for off-axis forces above a threshold of 10 $\mathrm{N}$. Once such a force is detected, the motor locks the position of the handpiece and real-time graphical feedback of the error is provided to the subject on a computer monitor. When the load cell detects appropriate forces towards the target, the graphical cue is removed and the motor unlocks the handpiece, allowing the subject to progress towards the target.

Our working hypothesis is that subjects will learn how to generate the correct muscle activations at troublesome workspace positions with this approach. If a subject repeats the proper muscle activations, they are rewarded with a smooth, uninterrupted movement to the target.

We addressed the issue of adapting the training to each subject's ability by adding a velocity-dependent assistive force from the motor. The difference between the maximum velocity of the previous reach and a desired velocity quantified as the maximum speed of a self-paced "comfortable" movement with the ipsilesional arm unattached to the device - determines a coefficient $b$ that specificies the assistive (or resistive) force for the next movement:

$$
b_{i}=b_{i-1}+c\left(\max \left(v_{i p s i}(t)\right)-\max \left(v_{i-1}(t)\right)\right)
$$

where $b_{i}$ is the velocity assistance coefficient for trial $i, c$ is an adaptation constant determining increment size, $v_{i-1}(t)$ is the velocity profile of the previous trial, and $v_{i p s i}(\mathrm{t})$ is the velocity profile of the ipsilesional arm. During trial $i$, the coefficient is multiplied by the instantaneous velocity to determine motor output:

$$
m_{i}(t)=b_{i} v_{i}(t)
$$

Thus, the motor does not assist movement unless the patient initiates movement. The assistance provided acts to destabilize the subject's movement, increasing the range and speed.

As the motor assistance increases the subject's movement velocity, the algorithm lowers the velocity assistance coefficient $b_{i}$ from trial to trial until the actual and desired peak movement speeds are equal. In the case that the user is able to move faster than the desired velocity, the algorithm decreases the velocity assistance eventually resisting movement if $b_{i}$ becomes negative. The algorithm is compatible with the guided force training because the assistance is velocity-dependent. Thus, if the subject's movement is stopped because of an errant off-axis force, then the assistance is terminated. When the subject generates forces in the correct direction, the assistance turns on smoothly.

\section{RESULTS}

\section{A. Convergence and repeatability of adaptive assistance}

The coefficient of assistance was found to converge to a consistent value for three subjects for reaching to a given target. As shown in Fig. 2, the coefficient for a severely impaired stroke subject CL01 repeatedly approached similar values on multiple visits to the lab when reaching to the same target. Furthermore, a lower value was approached for subject AL01, who also displayed greater functional ability on a clinical scale (3 out of 7 on the Chedoke-McMaster Stroke Assessment Scale [7]) compared with that for CL01 ( 2 out of 7). The coefficient became negative for an uninjured subject who was able to move at a velocity greater than the desired velocity. The gradual slope and late plateau for the unimpaired subject are due to the desired velocity for this subject being set to be relatively fast and the adaptation constant $c$ in (1) being designed for subjects with hemiparesis. The desired effect of allowing the subject to gradually reach faster and farther by increasing assistance is confirmed in Fig. 3.

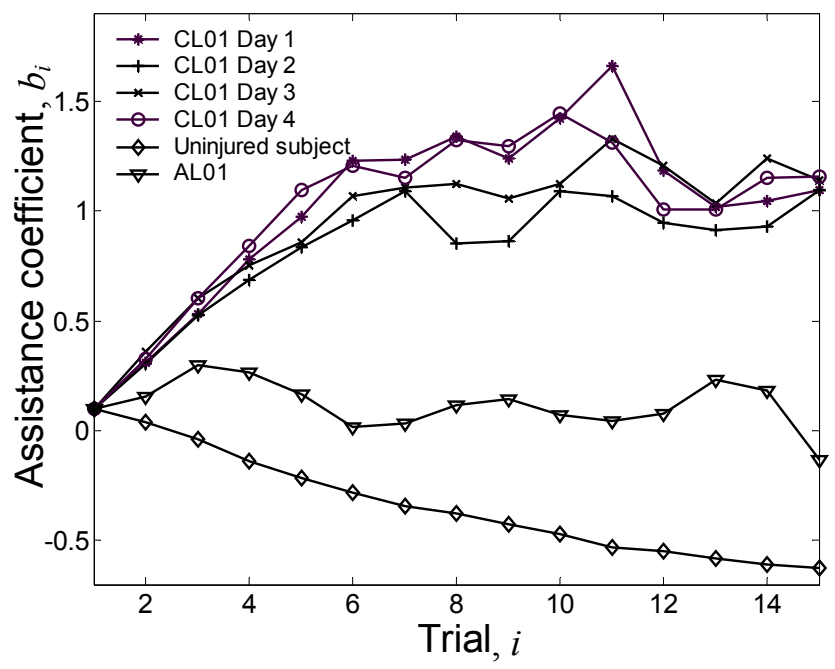

Fig. 2. Convergence of the assistance over multiple trials. Values for stroke subject CL01 consistently converged around a single value $(\sim 1.1)$ over multiple sessions on different days. Values for a single session are also shown for stroke subject AL01 and an uninjured subject. 


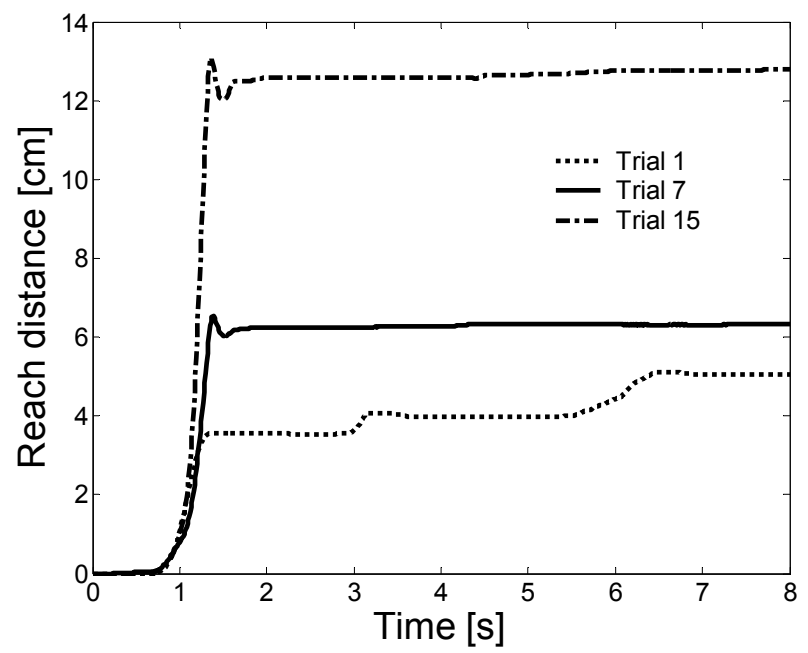

Fig. 3. Increasing assistance allows a user to reach farther. Trajectories shown are from trials 1, 7, and 15 on day 4 from subject CL01 (see Fig. 2).

\section{B. Therapeutic outcomes}

To date, one subject with hemiparesis has completed eight weeks of guided force training with the ARM Guide. The subject exhibited significant increases in reaching range of motion $(\mathrm{p}<0.025)$ and maximum velocity $(\mathrm{p}<0.001)$ along the ARM Guide according to a two-sample t-test (Table I). These were measured by disabling the motor and asking subjects to reach as far and as fast as possible. Improvement was also seen as a decrease in the mean time per task to complete four functional activities of daily living (ADLs): clearing the impaired arm during shirt tuck, stabilizing a pillow, stabilizing a small package, and wringing a rag.

TABLE I

OUTCOMES OF CONTRALESIONAL ARM FUNCTION BEFORE AND AFTER TRAINING

\begin{tabular}{cccccc}
\hline $\begin{array}{c}\text { Normalized reach } \\
\text { distance }\end{array}$ & \multicolumn{2}{c}{$\begin{array}{c}\text { Normalized reach } \\
\text { velocity }\end{array}$} \\
$\begin{array}{c}\text { [\% of ipsilesional } \\
\text { reach distance }]\end{array}$ & $\begin{array}{c}\text { A\% of ipsilesional } \\
\text { velocity] }\end{array}$ & $\begin{array}{c}\text { Avg. time to complete } \\
\text { 4 functional tasks [s] }\end{array}$ \\
\hline Pre & Post & Pre & Post & Pre & Post \\
\hline 6.9 & 16.7 & 6.9 & 16.3 & 36.3 & 25.8 \\
\hline
\end{tabular}

\section{DiscusSiON}

This paper describes the design and initial testing of a novel robotic training algorithm that combines guided force training and adaptive assistance. The assistive controller works by making the movement less stable, but, because the forces it provides are small compared to the net endpoint force needed to move the limb, the technique supplements the user-generated acceleration and deceleration force rather than creating rapid, unstable accelerations. Limits were set on the increment size of $b$ from one trial to another in addition to other redundant safety mechanisms. As confirmed in Fig. 3, the assistance gradually adapted to a level that allowed the subject to reach farther and faster, and thus achieve a more normative movement.

We have just begun a study to examine the efficacy of this training in restoring volitional arm function in patients with severe hemiparesis. Twenty subjects will participate in eight weeks of guided force training and their outcomes will be compared to twenty subjects participating in a matched amount of conventional therapy and twenty subjects participating in a matched amount of free (without the device) reaching practice.

\section{CONCLUSION}

The methods described demonstrate the feasibility of a guided force training paradigm with adaptive assistance. This form of training for the hemiparetic upper extremity can be effectively carried out with a velocity-dependent assistance that requires volitional effort from the user, adapts to each subject's ability, and allows even more severely impaired subjects to complete the training task. Early results from a single subject indicate that the desired effect of improved arm function can be achieved with guided force training.

\section{ACKNOWLEDGMENT}

This study was supported by a Rehabilitation Engineering Research Center grant (H133E020724) from NIDRR.

\section{REFERENCES}

[1] S. E. Fasoli, H. I. Krebs, J. Stein, W. R. Frontera, and N. Hogan, "Effects of robotic therapy on motor impairment and recovery in chronic stroke," Archives of Physical Medicine \& Rehabilitation, vol. 84, pp. 477-482, 2003.

S. Hesse, G. Schulte-Tigges, M. Konrad, A. Bardeleben, and C. Werner, "Robot-assisted arm trainer for the passive and active practice of bilateral forearm and wrist movements in hemiparetic subjects," Archives of Physical Medicine \& Rehabilitation, vol. 84, pp. 915-920, 2003.

[3] D. J. Reinkensmeyer, L. E. Kahn, M. Averbuch, A. McKenna-Cole, B. D. Schmit, and W. Z. Rymer, "Understanding and treating arm movement impairment after chronic brain injury: Progress with the ARM Guide," Journal of Rehabilitation Research and Development, vol. 37, pp. 653-662, 2000. 
[4] P. S. Lum, C. G. Burgar, P. C. Shor, M.

Majmundar, and M. Van der Loos, "Robot-assisted movement training compared with conventional therapy techniques for the rehabilitation of upperlimb motor function after stroke," Archives of Physical Medicine \& Rehabilitation, vol. 83, pp. 952-9, 2002.

[5] M. L. Aisen, H. I. Krebs, N. Hogan, F. McDowell, and B. T. Volpe, "The effect of robot-assisted therapy and rehabilitative training on motor recovery following stroke," Archives of Neurology, vol. 54, pp. 443-6, 1997.

[6] L. E. Kahn, P. S. Lum, and D. J. Reinkensmeyer, "Selection of robotic therapy algorithms for the upper extremity in chronic stroke: Insights from MIME and ARM Guide results," presented at International Conference on Rehabilitation

Robotics, Daejeon, Korea, 2003.

[7] C. Gowland, P. Stratford, M. Ward, J. Moreland, W. Torresin, S. Van Hullenaar, J. Sanford, S.

Barreca, B. Vanspall, and N. Plews, "Measuring physical impairment and disability with the Chedoke-McMaster Stroke Assessment," Stroke, vol. 24, pp. 58-63., 1993. 